\title{
A Modified Energy Efficient Multi-Hop Routing Protocol in Wireless Sensor Networks
}

\author{
A. D. Usman*, F. S. Dadah, S. M. Sani \\ Department of Communications Engineering, Ahmadu Bello University, Zaria, Kaduna State, Nigeria.
}

\begin{abstract}
Energy efficient routing protocols in Wireless Sensor Networks (WSNs) is an important area of research due to energy limitations. It is therefore important to maximize the limited energy so as to increase the network lifetime of the WSN. In this paper, a modified energy efficient multi-hop routing protocol (mEEMRP) in a $200 \mathrm{~m}^{2}$ field is presented. This protocol is based on a technique that involves balancing load between communication management (CM) nodes during the multi-hop routing of aggregated data to the base station (BS), where the residual energy (RE) levels of CM nodes are considered as well as the distance between neighboring CM nodes. Simulation results showed that mEEMRP yielded a 1.77\% improvement over energy efficient multi-hop routing protocol (EEMRP) in terms of network lifetime. More so, the proposed mEEMRP also improved the energy consumption and the number of packets received at the BS by $4.83 \%$ and $7.41 \%$, respectively.
\end{abstract}

KEYWORDS: Routing protocol, Multi-hop routing, Network lifetime, mEEMRP, EEMRP.

[Received March 01, 2018; Revised April 23, 2018; Accepted May 01, 2018]

Print ISSN: 0189-9546 | Online ISSN: 2437-2110

\section{INTRODUCTION}

A WSN is a network that is composed of hundreds of sensor devices that communicate over wireless channels (Vijayan \& Raaza, 2016). With recent advances in microelectromechanical-systems (MEMS) (MEMS are microscopic devices that have moving parts and consist of a microprocessor and several components that interact with the surrounding such as microsensors.) and wireless communication, WSN technologies provide a wide range of advantages over conventional networking technologies (Rawat et al., 2014). Some of these advantages include lower cost, scalability, reliability, accuracy, flexibility and ease of deployment. Areas of application of WSNs include military, healthcare systems, security, surveillance and agriculture (Rawat et al., 2014). The communication in WSNs is governed by unique set of rules called routing protocols (Vijayan \& Raaza, 2016).

The sensor nodes (SNs) transmit their sensed data through inbuilt transmitters as electromagnetic signals to a designated BS either directly (Akkaya \& Younis, 2005) or through intermediate nodes (Gupta et al., 2017). WSNs are often deployed in areas or regions that are not easily accessible (Jan et al., 2013). Batteries are therefore not easily replaced or recharged. The limited energy of the SNs and the inaccessibility of the regions where they are deployed necessitate the need for energy conservation in the sensor nodes in order to maximize network lifetime and ensure that the network is not partitioned (Gupta et al., 2017). This is in order to avoid sensor voids or holes (More \& Raisinghani, 2016).

In this research paper, a load balancing technique was used. This technique maintained load balance among CMs nodes during the routing of data to the BS. This was done in order to increase the network lifetime of the WSN, minimize the energy consumption of SNs and increase the number of packets received at the BS.

\section{REVIEW OF SIMILAR WORKS}

A lot of research has been carried out in the area of energy efficiency in WSNs. Another major limitation in WSN deployment is the problem of coverage area. Deploying more SNs within the WSN to provide more coverage is generally not a viable solution to the coverage area problem. This is due to increase in radio interference as well as deployment and maintenance cost (Vecchio \& Lopez-Valcarce, 2015). Presented below are some literatures highlighting the techniques adopted in utilizing the limited energy of SNs in order to maximize network lifetime.

Mahmood et al., (2013) proposed a modified LEACH (MODLEACH) by introducing an efficient cluster head (CH) replacement scheme and dual transmitting power levels. In the proposed protocol, a threshold was used in determining whether a $\mathrm{CH}$ could serve as $\mathrm{CH}$ for the next round or had to be replaced. A $\mathrm{CH}$ would only be replaced when its energy level fell below a certain threshold, thereby minimizing the overhead of the protocol, which in turn reduced the energy that would have been expended in selecting new $\mathrm{CHs}$ and creating new clusters. Furthermore, the protocol utilized dual transmitting power levels to amplify transmitted signals according to the nature of the transmission; intra cluster transmission, inter cluster transmission and $\mathrm{CH}$ to $\mathrm{BS}$ transmission.

Finally, hard and soft thresholds were implemented on MODLEACH to give comparison on the performance of the protocol. The proposed protocol was evaluated using $\mathrm{CH}$ formation, throughput and network lifetime as performance 
metrics and MODLEACH showed significant improvements in all performance metrics. However, by extending the number of rounds a node could serve as $\mathrm{CH}$, this could result in such a node dying quickly because it had to use its energy to a certain level before it stopped serving as $\mathrm{CH}$. This scenario gives rise to uneven distribution of energy throughout the network as some nodes will have higher residual energy levels compared to other nodes that are serving as $\mathrm{CH}$.

Guiloufi et al., (2014) proposed an energy efficient clustering algorithm (EECA) for fixed and mobile SNs by considering three scenarios; fixed nodes (EECA-F), constant mobility nodes (EECA-M1) and dynamic mobility nodes (EECA-M2). In all three scenarios, the respective protocols considered node degree, consumed energy of node and distance of the node from the BS. These parameters were used in calculating weights for every SN. In EECA-M1, three ranges of speed were defined; $1(0-5 \mathrm{~km} / \mathrm{h}), 2(5-20 \mathrm{~km} / \mathrm{h})$ and $3(20-44 \mathrm{~km} / \mathrm{h})$. The speed of a SN belonged to one of these groups and was therefore considered constant. For EECAM2, node mobility (Mob(u)) was also used in determining the weights for the SNs. The node with the least weight, which represented the node with maximum degree, minimum consumed energy and closest to the BS, was elected as the $\mathrm{CH}$. Results showed improvement in energy saving of sensor nodes by reducing the power consumption of nodes. However, this protocol leads to increased overhead as a result of the different scenarios in which it operates. This increased complexity will further lead to considerable loss of energy which will lead to nodes dying quicker.

Zahedi et al., (2015) conducted an experimental measurement and analysis of electromagnetic communication in Underwater Wireless Sensor Networks (UWSN). It was established that acoustic signals were highly affected by several challenges. Some of these challenges include low propagation speed, low bandwidth and ambient noise. Electromagnetic signals were then used to replace acoustic signals for UWSN. The results for two measurements in freshwater and seawater for UWSN at $24 \mathrm{GHz}$ were presented. Results obtained showed that the maximum communication range in freahwater was $14.4 \mathrm{~cm}$ for $10 \mathrm{~cm}$ deployment depth while the maximum communication range for seawater was $5 \mathrm{~cm}$ for $2 \mathrm{~cm}$ deployment depth. Both measurement results show short communication ranges. As a result of these observations, a correction factor was incorporated in the general path loss prediction model for underwater electromagnetic communication.

Gwavava \& Ramanaiah, (2015) proposed yet another LEACH (YA-LEACH), a WSN routing protocol that used centralized cluster formation to ensure optimal clusters and allow $\mathrm{CHs}$ to extend operation into multiple rounds to achieve energy savings. The protocol used an alternative (vice) $\mathrm{CH}$ that took over the role of $\mathrm{CH}$ when the residual energy of the $\mathrm{CH}$ was not enough to last the entire round. The centralized clustering scheme ensured fair distribution of $\mathrm{CHs}$ throughout the network. A $\mathrm{CH}$ was allowed to extend its role into another round provided it had enough residual energy.
This residual energy was calculated as the minimum energy required before the $\mathrm{CH}$ has to transfer its role to the vice- $\mathrm{CH}$. By so doing, the proposed protocol saved considerable amounts of setup cost as clustering did not have to be done after every round. Simulation results showed an increase in terms of network lifetime and data throughput. However, by extending the number of rounds a $\mathrm{CH}$ remained as $\mathrm{CH}$, there could be uneven distribution of energy throughout the network as $\mathrm{CHs}$ have to use up almost all of their energy before relinquishing their roles as CHs. This could also lead to such nodes dying quicker and resulting in sensor holes in the network.

Ke et al., (2016) proposed a novel energy aware hierarchical cluster-based (NEAHC) routing protocol to minimize total energy consumption and ensure fairness of energy consumption between nodes. In the proposed protocol, $\mathrm{CHs}$ were selected on the basis of remaining energy. Nodes with low energy levels switch between sleep and active modes in order to balance energy consumption. Relay node choosing problem was modeled as a nonlinear programming problem and the property of convex function was used to find the optimal solution. Simulation results showed there was significant improvement in terms of network lifetime, energy consumption per round and reliable delivery of data. However, the proposed protocol does not ensure even distribution of $\mathrm{CHs}$ throughout the network as $\mathrm{CHs}$ were chosen based on remaining energy alone. This can lead to uneven energy distribution in the WSN since CHs were not efficiently distributed throughout the network.

Singh \& Verma, (2017) proposed an energy efficient cross layer based adaptive threshold routing protocol for WSNs. The proposed protocol was proposed for networks which are heterogeneous and was based on adaptive threshold sensitive distributed energy efficient cross layer routing protocol. The principle of weights was used in selecting $\mathrm{CHs;}$ a ratio of the average energy of the entire network and the residual energy of the SN. The protocol also incorporated reactive and proactive network concepts. Simulation results showed that the proposed protocol outperforms other protocols in the area of data packets received at the BS, network residual energy and the number of nodes alive. However, the proposed protocol does not lead to optimal distribution of $\mathrm{CHs}$ in the network since $\mathrm{SNs}$ with high residual energy could be located in one part of the network. This will mean more $\mathrm{CHs}$ will be selected from that part of the network causing other SNs to do more work in transmitting data to their CHs. This will result in uneven distribution of energy load in the network.

Huang et al., (2017) proposed an energy efficient multihop routing protocol (EEMRP) based on grid clustering to tackle the problem of unbalanced energy consumption of SNs. In the protocol, the network area was divided into unequal grids which formed different levels of clusters. In order to minimize the energy consumption of nodes, the protocol optimized the process of electing communication management (CM) nodes and $\mathrm{CHs}$ by combining nodes' energy level, location and levels of the network. A SN that 
had a higher RE and was located closer to the BS in each grid had a higher probability to become a CM node or $\mathrm{CH}$. A shortest path multi-hop routing algorithm was adopted in routing aggregated data to the BS. Simulation results showed an extension in network lifetime and better performance of energy balance and efficiency in larger network areas when compared to other routing protocols. However, with increase in rounds and disparity in energy levels in the network, multihop routing on the basis of distance alone could lead to CM nodes with higher RE levels being neglected as next hops. This can further lead to uneven energy levels and unbalanced energy consumption among the CM nodes in the network which can result in reduction in network lifetime in terms of FND.

It can be seen from the literatures reviewed that energy efficient routing in WSNs is important as it provides a way of utilizing the limited energy of SNs. This paper presents a modified energy efficient multi-hop routing (mEEMRP) that seeks to balance load between CM nodes.

\section{METHODOLOGY}

This research work was carried out by replicating the EEMRP of Huang et al., (2017). This is done by dividing the network area into grids, with each grid identified by a tuple $(\mathrm{u}, \mathrm{v})$ where the tuple represents the vth grid of the uth lane. The SNs in the WSN were randomly deployed in the field. The random deployment of nodes was adopted since practical WSN deployments are often random in nature.

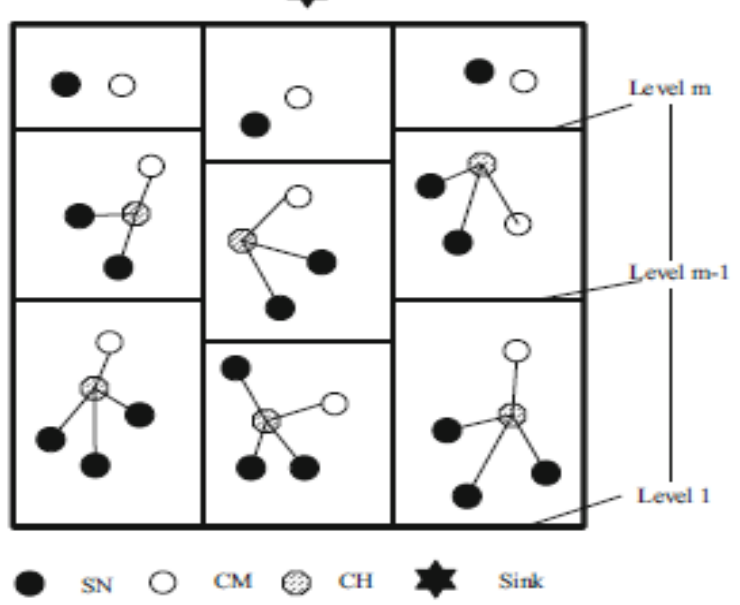

Figure 1: Network Area Division into Grids (Huang et al., 2017).

A centralized $\mathrm{CM}$ election algorithm was used by the BS in selecting CM nodes based on a weight formula (Huang et al., 2017):

$$
\mathrm{W}_{\mathrm{i}}^{0}=\frac{\mathrm{E}_{\mathrm{i}}(\mathrm{t})-\alpha \times \mathrm{E}_{\mathrm{r}}(\mathrm{t}) a v}{\alpha \times \mathrm{E}_{\mathrm{r}}(\mathrm{t}) a v} \times \frac{\mathrm{d}_{\mathrm{av}}}{\mathrm{d}_{\left(\mathrm{s}_{i}, \mathrm{~s}_{0}\right)}}
$$

if $\mathrm{E}_{\mathrm{i}}(\mathrm{t})>\alpha \times \mathrm{E}_{\mathrm{r}}(\mathrm{t})_{\mathrm{av}}$

where:

$\mathrm{E}_{\mathrm{i}}(\mathrm{t})$ is the residual energy of the $\mathrm{i}$-th node

$E_{r}(t)_{a v}$ is the average residual energy of the network in round $\mathrm{r}$ $\alpha$ is the weighted coefficient of SNs, $0<\alpha \leq 1$

$\mathrm{d}\left(\mathrm{s}_{\mathrm{i}}, \mathrm{S}_{0}\right)$ is the distance between SN $\mathrm{i}$ and the BS

$\mathrm{d}_{\mathrm{av}}$ is the average distance between SNs and the BS

The CM node is responsible for routing aggregated data to the BS while the $\mathrm{CH}$ is responsible for aggregating sensed data from individual SNs within the grid. Aggregated data from $\mathrm{CHs}$ are forwarded to the $\mathrm{BS}$ via multiple hops when the distance between the transmitting CM node and the BS is large. The multi-hop route is selected based on the shortest path between a transmitting $\mathrm{CM}$ node and its neighboring $\mathrm{CM}$ nodes.

The modified energy efficient multi-hop routing protocol (mEEMRP) presented in this paper is realized by adopting a threshold that determined which CM node would be selected as next hop during the multi-hop routing of aggregated data to the BS. This technique takes into consideration the RE of neighboring CM nodes as well as the distance between the neighboring $\mathrm{CM}$ nodes. This technique ensures even distribution of energy levels throughout the WSN. This is achieved by selecting a next hop CM node as the CM node with the highest RE and within the distance threshold, $\mathrm{d}_{0}$.

The distance threshold, $\mathrm{d}_{0}$, is as specified in the work of Huang et al., (2017) and is given by the equation (Huang et al., 2017):

$$
\mathrm{d}_{0}=\sqrt{\frac{E_{\mathrm{amp} 1}}{\mathrm{E}_{\mathrm{amp} 2}}}
$$

where:

$\mathrm{d}_{0}$ is the distance threshold

$\mathrm{E}_{\text {ampl }}$ is the amplifier transmitter dissipation if $\mathrm{d}<\mathrm{d}_{0}$ which is $10 \mathrm{pJ} / \mathrm{bit} / \mathrm{m}^{2}$

$E_{\text {amp2 }}$ is the amplifier transmitter dissipation if $d \geq d_{0}$ which is $0.0013 \mathrm{pJ} / \mathrm{bit} / \mathrm{m}^{2}$

Figure 2 shows the representation of multi-hop communication where intermediate $\mathrm{CM}$ nodes serve as relay nodes to the BS.

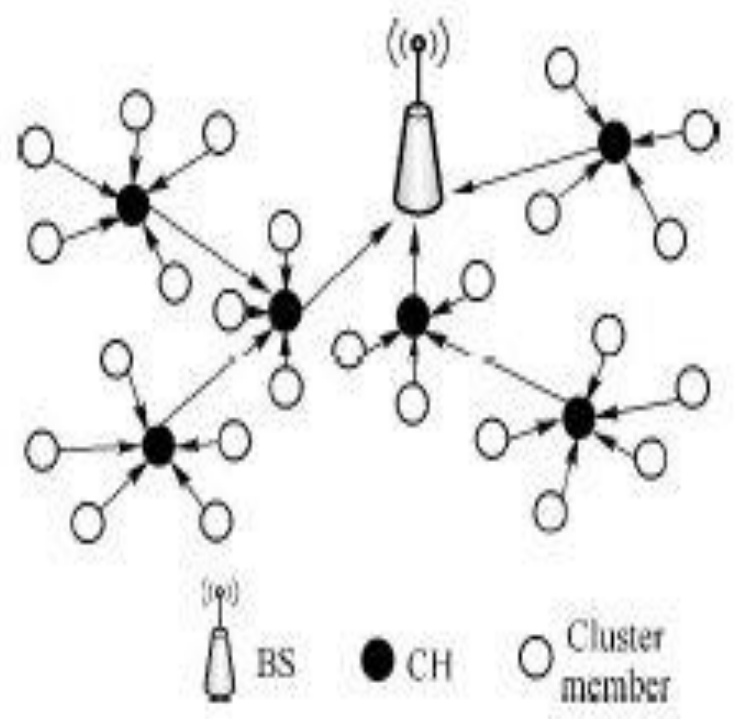

Figure 2: Network Topology formed by Multi-hop Communication (Ke et al., 2016). 
The following assumptions and parameters were used in carrying out simulations:

a. The location of the BS and all sensor nodes are fixed.

b. The deployment of sensor nodes was random.

c. The number of SNs deployed is 400 .

d. The location of the BS $(100,200)$ is known in advance.

e. Rectangle numbers is 4 .

f. Rectangle width is $50 \mathrm{~m}$

g. The data packet size is 800 bits.

h. Initial energy of SNs is $0.5 \mathrm{~J}$.

i. Grid numbers of each rectangle is: $\mathrm{A}=4,4,4,4$

$\mathrm{j}$. The network scenario is a $200 \mathrm{~m}$ by $200 \mathrm{~m}$ field

$\mathrm{k}$. distance threshold, $\mathrm{d}_{0}=87.7 \mathrm{~m}$

The flowchart of the mEEMRP is as shown in Figure 3.

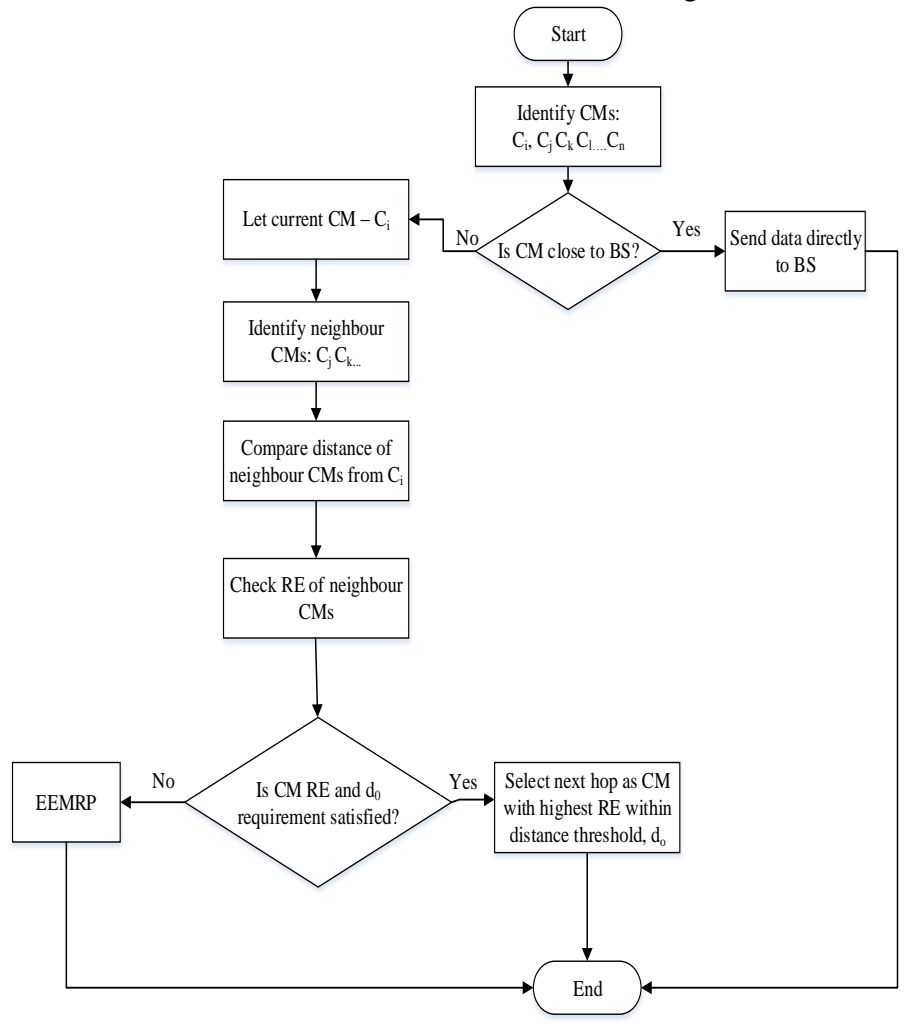

Figure 3: Flowchart of mEEMRP.

\section{RESULTS AND DISCUSSION}

The modified protocol was simulated and implemented on MATLAB R2015a simulator with the results obtained compared with EEMRP based on network lifetime, energy consumption percentage and number of packets received at the BS. The results obtained for each performance metric are presented thus:

\section{A. Result for Node Death Percentage (Network Lifetime)}

In Figure 4, node death percentage is plotted against rounds in a $200 \mathrm{~m}$ by $200 \mathrm{~m}$ network field containing 400 sensor nodes (SNs). The network lifetime is taken as the number of rounds it takes for $100 \%$ of the SNs in the network to die. From the figure, using EEMRP as routing protocol, it took 800 rounds for the first node in the network to die (referred to as first node death, FND) as against the 817 rounds it took the mEEMRP routing protocol. Also, using EEMRP, the last node in the network died at 849 rounds (referred to as last node death, LND) as against 864 rounds using mEEMRP. This indicates that by considering residual energy levels of next hop CM nodes as well as the distance between CM nodes, the network lifetime was extended.

The result in Figure 4 for both routing protocols follow a similar pattern because both mEEMRP and EEMRP use the same CM election algorithm and similar multi-hop routing scheme with the difference coming in the inclusion of the energy factor during the multi-hop routing in mEEMRP. It can thus be said that by using mEEMRP, the network lifetime is $2.12 \%$ and $1.77 \%$ longer than the EEMRP in terms of first node death and last node death respectively.

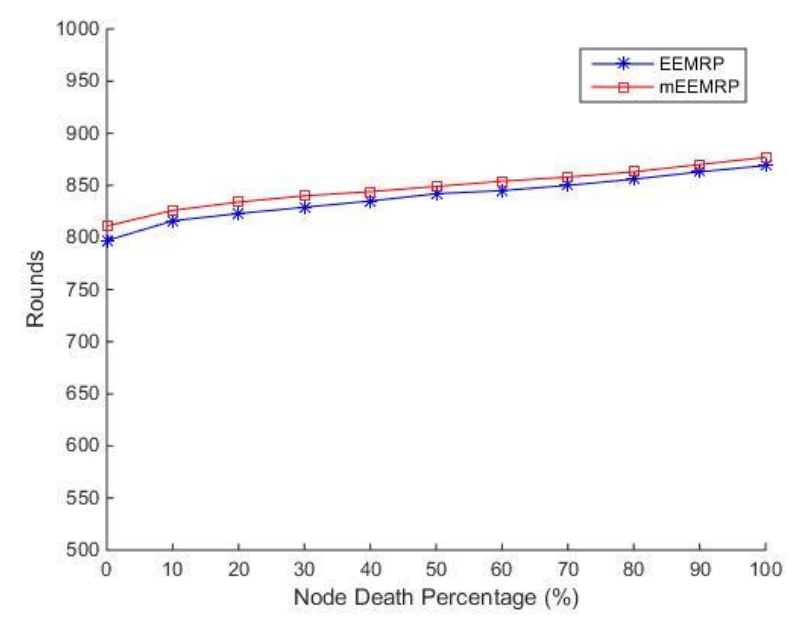

Figure 4: Node Death Percentage Plot.

The percentage improvements presented in Table 1 represent significant improvements in terms of network lifetime with an increase of 17 rounds in terms of FND and 15 rounds in terms of LND.

Table 1: Summary of Results on Node Death Percentage.

\begin{tabular}{llll}
\hline Network & EEMRP & mEEMRP & $\begin{array}{l}\text { Percentage } \\
\text { Improvement }\end{array}$ \\
Lifetime & & & \\
\hline FND & 800 & 817 & $2.12 \%$ \\
LND & 849 & 864 & $1.77 \%$ \\
\hline
\end{tabular}




\section{B. Result for Energy Consumption Percentage}

In Figure 5, energy consumption percentage is plotted against the number of rounds in a $200 m$ by $200 m$ network field containing $400 \mathrm{SNs}$. From the figure, it can be seen that the energy consumption pattern for both routing protocols follow a similar trend. As the number of rounds increase the amount of energy used in the network increases, hence an increase in the energy consumption percentage. Using EEMRP, it can be seen that the energy consumption percentage at different intervals correspond to number of rounds that are considerably less than that of their corresponding energy consumption percentage intervals of mEEMRP. This shows that mEEMRP has a better energy consumption percentage over EEMRP as the residual energy levels of CM nodes are taken into account as well as their distances during the multi-hop routing of data. From the results obtained, it can be stated that mEEMRP improved the energy consumption percentage by $4.83 \%$ over the EEMRP.

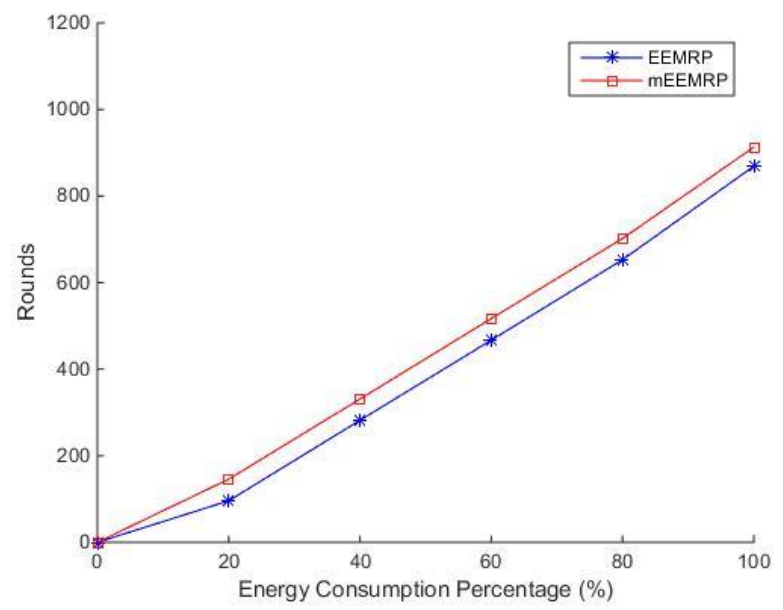

Figure 5: Energy Consumption Percentage Plot.

\section{Result for Number of Packets Delivered at the Base Station}

In Figure 6, number of packets delivered at the BS is plotted against number of rounds in a $200 \mathrm{~m}$ by $200 \mathrm{~m}$ network field containing $400 \mathrm{SNs}$. The number of packets delivered to the BS refers to the number of packets that are successfully received at the designated BS. From the figure, as the number of rounds increase the number of packets delivered at the BS increases. Using EEMRP as routing protocol, it can be seen that the number of packets delivered at the $\mathrm{BS}$ at different rounds are considerably less the number of packets delivered at the BS for corresponding intervals for the mEEMRP. This shows that mEEMRP performs better than EEMRP in terms of the number of packets delivered to the BS at different rounds. From the results obtained, it can be stated that mEEMRP performs better than EEMRP by $7.41 \%$.

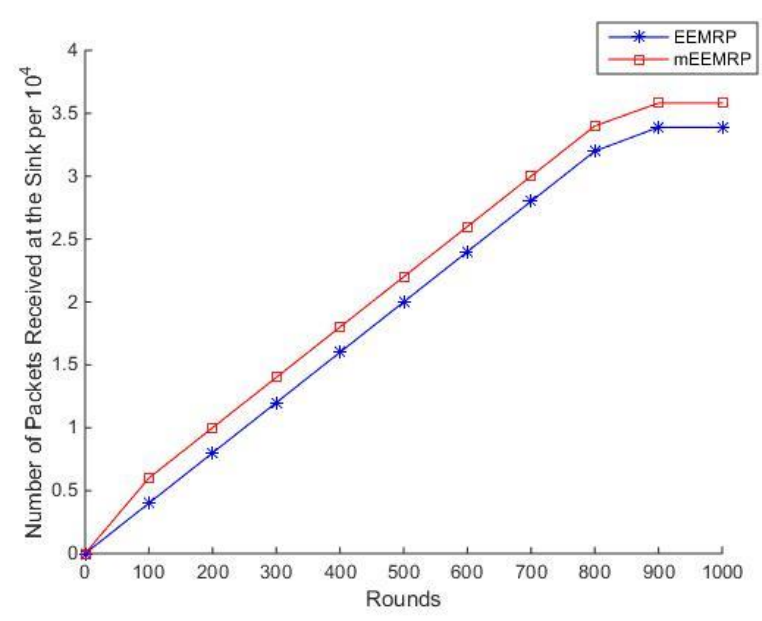

Figure 6: Number of Packets Received at the BS Plot.

\section{CONCLUSION}

In this paper, a modified energy efficient multi-hop routing protocol (mEEMRP) was presented. In the protocol, $\mathrm{CM}$ nodes in grids that are located close to the BS send aggregated data to the BS directly while CM nodes in grids that are far away from the BS send their aggregated data via multi-hop transmission. The developed protocol increased the network lifetime and improved on the energy consumption percentage as well as the number of packets received at the BS. As a recommendation for future work, the grid regions can be optimized to ensure reliable communication between clusters and to further improve the network performance. This routing protocol can be extended to larger network regions to test its scalability.

\section{ACKNOWLEDGEMENTS}

This work was supported by the Department of Communications Engineering, Ahmadu Bello University, Zaria. The authors are also grateful to the anonymous referees for their insightful and valuable suggestions and contributions.

\section{REFERENCES}

Akkaya, K., \& Younis, M. (2005). A survey on routing protocols for wireless sensor networks. Ad hoc networks, 3(3): 325-349.

Guiloufi, A. B., N., Nasri, \& A., Kachouri (2014). Energy-efficient clustering algorithms for fixed and mobile Wireless Sensor Networks. Paper presented at the 2014 International Wireless Communications and Mobile Computing Conference (IWCMC). Nicosia, Cyprus, 735-738.

Gupta, S. K., P., Kuila, \& P. K., Jana(2017). GA Based Energy Efficient and Balanced Routing in k-Connected Wireless Sensor Networks. Paper presented at the Proceedings of the First International Conference on Intelligent Computing and Communication. Singapore, 679686.

Gwavava, W., \& Ramanaiah, O. (2015). YA-LEACH: Yet another LEACH for wireless sensor networks. Paper 
presented at the 2015 International Conference on Information Processing (ICIP). Pune, India, 96-101.

Huang, J.; Y. Hong, Z. Zhao, \& Y. Yuan, (2017). An energy-efficient multi-hop routing protocol based on grid clustering for wireless sensor networks. Cluster Computing, 20(4): 3071-3083.

Jan, M. A.; P., Nanda, X., He, \& R. P., Liu (2013). Enhancing lifetime and quality of data in cluster-based hierarchical routing protocol for wireless sensor network. Paper presented at the 2013 IEEE International Conference on High Performance Computing and Communications (HPCC) \& 2013 IEEE International Conference on Embedded and Ubiquitous Computing (EUC), Zhangjiajie, Hunan Province, P.R. China, $1400-1407$.

Ke, W.; O. Yangrui, J. Hong, Z. Heli and L. Xi, (2016). Energy aware hierarchical cluster-based routing protocol for WSNs. The Journal of China Universities of Posts and Telecommunications, 23(4), 46-52.

Mahmood, D.; N., Javaid, S., Mahmood, S., Qureshi, A. M., Memon, \& T., Zaman (2013). MODLEACH: a variant of LEACH for WSNs. Paper presented at the 2013 Eighth International Conference on Broadband and Wireless Computing, Communication and Applications (BWCCA). Karachi, Pakistan, 158-163.

More, A., \& Raisinghani, V. (2017). A survey on energy efficient coverage protocols in wireless sensor networks. Journal of King Saud University-Computer and Information Sciences, 29(4): 428-448.

Rawat, P., K. D., Singh, H., Chaouchi, \& J. M. Bonnin (2014). Wireless sensor networks: a survey on recent developments and potential synergies. The Journal of supercomputing, 68(1), pp 1-48.

Singh, R., \& Verma, A. K. (2017). Energy efficient cross layer based adaptive threshold routing protocol for WSN. AEU-International Journal of Electronics and Communications, 72, pp 166-173.

Vecchio, M., \& López-Valcarce, R. (2015). Improving area coverage of wireless sensor networks via controllable mobile nodes: A greedy approach. Journal of network and computer applications, 48, 1-13.

Vijayan, K., \& Raaza, A. (2016). A novel cluster arrangement energy efficient routing protocol for wireless sensor networks. Indian Journal of science and Technology, 9(2): $1-9$.

Zahedi, Y.; R., Ngah, A. Y., Abdulrahman, M., Mokayef, S. E., Alavi, K., Zahedi, \& S. H., Arrifin (2015). Experimental measurement and analysis of electromagnetic communication in underwater wireless sensor networks. Journal of Computational and Theoretical Nanoscience, 12(12), 6069-6076. 